\title{
Phonon surface mapping of graphite: Disentangling quasi-degenerate phonon dispersions
}

\author{
A. Grüneis, ${ }^{1,2}$ J. Serrano, ${ }^{3}$ A. Bosak, ${ }^{4}$ M. Lazzeri, ${ }^{5}$ S. L. Molodtsov,${ }^{6}$ L. Wirtz,${ }^{7}$ C. Attaccalite,${ }^{8}$ M. Krisch, ${ }^{4}$ A. Rubio,${ }^{8,9}$ \\ F. Mauri, ${ }^{5}$ and T. Pichler ${ }^{1}$ \\ ${ }^{1}$ Faculty of Physics, University of Vienna, Boltzmanngasse 5, A-1090 Vienna, Austria \\ ${ }^{2}$ IFW-Dresden, P.O. Box 270116, D-01171 Dresden, Germany \\ ${ }^{3}$ ICREA-Departamento de Fisica Aplicada, Universitat Politecnica de Catalunya, EPSC, Av. Esteve Terradas 15, \\ 08860 Castelldefels, Spain \\ ${ }^{4}$ European Synchrotron Radiation Facility, BP 220, F-38043 Grenoble Cedex, France \\ ${ }^{5} I M P M C, I P G P$, Universités Paris 6 et 7, CNRS, 140 rue de Lourmel, 75015 Paris, France \\ ${ }^{6}$ Institut für Festkörperphysik, TU Dresden, Mommsenstrasse 13, D-01069 Dresden, Germany \\ ${ }^{7}$ Institute for Electronics, Microelectronics, and Nanotechnology (CNRS UMR 8520), Dept. ISEN, B.P. 60069, \\ 59652 Villeneuve d'Ascq Cedex, France \\ ${ }^{8}$ Nano-Bio Spectroscopy Group and ETSF Scientific Development Centre, Dpto. Física de Materiales, Universidad del País Vasco, \\ Centro de Física de Materiales CSIC-UPV/EHU-MPC and DIPC, Av. Tolosa 72, E-20018 San Sebastián, Spain \\ ${ }^{9}$ Fritz-Haber-Institute of the Max Planck Society, Theory Department, Faradayweg 4-6, D-14195 Berlin-Dahlem, Germany
}

(Received 22 July 2009; published 19 August 2009)

\begin{abstract}
The two-dimensional mapping of the phonon dispersions around the $K$ point of graphite by inelastic x-ray scattering is provided. The present work resolves the longstanding issue related to the correct assignment of transverse and longitudinal phonon branches at $K$. We observe an almost degeneracy of the three TO-, LA-, and LO-derived phonon branches and a strong phonon trigonal warping. Correlation effects renormalize the Kohn anomaly of the TO mode, which exhibits a trigonal warping effect opposite to that of the electronic band structure. We determined the electron-phonon coupling constant to be $166(\mathrm{eV} / \AA)^{2}$ in excellent agreement to GW calculations. These results are fundamental for understanding angle-resolved photoemission, doubleresonance Raman and transport measurements of graphene-based systems.
\end{abstract}

DOI: 10.1103/PhysRevB.80.085423

PACS number(s): 71.38.-k, 71.10.-w, 79.60.-i

\section{INTRODUCTION}

The lattice dynamics of most "standard" materials are well known nowadays and can be routinely described by $a b$ initio methods based on density-functional perturbation theory. ${ }^{1}$ The phonon-dispersion relations of graphite and graphene have already been determined by inelastic neutron scattering, ${ }^{2}$ inelastic $x$-ray scattering (IXS), ${ }^{3,4}$ doubleresonance Raman scattering, 5,6 and electron-energy-loss spectroscopy. ${ }^{7}$ After the first $a b$ initio calculations ${ }^{8,9}$ had been corroborated by IXS, ${ }^{3,4,10}$ the story for graphite seemed closed. ${ }^{11}$ However, the phonon dispersions of graphite and graphene-a single layer of graphite-have continued to present surprises in recent years: (i) two Kohn anomalies ${ }^{12}$ at the Brillouin-zone $(\mathrm{BZ})$ center $(\Gamma)$ and corner $(K)$ were predicted theoretically ${ }^{13,14}$ and (ii) for charged graphene/ graphite, the (electronic) nonadiabatic effects at $\Gamma$ were predicted theoretically ${ }^{15}$ and confirmed experimentally by Raman scattering. ${ }^{16,17}$ Very similar observations have been made for carbon nanotubes. ${ }^{18-22}$ The precise understanding of the Kohn anomaly is highly significant for electronphonon coupling (EPC) and thus of prime importance for the quantitative description of superconductivity in graphite intercalation compounds, ${ }^{23,24}$ electronic transport in the highbias regime, and for the (double) resonant Raman scattering. ${ }^{5,25}$ The latter is now commonly used to distinguish single-layer graphene from double-layer and multilayer graphene. ${ }^{26,27}$

Qualitatively, the Kohn anomaly of the transversal-optical (TO) branch at the $K$ point is well described by density- functional theory (DFT) ${ }^{14}$ However, its magnitude is severely underestimated by DFT. ${ }^{28,29}$ Electron-electron correlation leads to an enhancement of the EPC at $K$. This can be quantitatively calculated ${ }^{29}$ with the GW approximation (Green's function G of the screened Coulomb interaction W) which also gives very good results for the electronic band structure of graphite (see, e.g., Ref. 30). Although the EPC at $\Gamma$ can be derived from the IXS and Raman linewidth measurements ${ }^{31}$ the experimental determination of the EPC close to the $K$ point $^{29}$ is still missing. The experiments needed to unravel the details of the phonon dispersions are extremely challenging because three phonon branches are overlapping in a small energy window and the phonon energies have a strong dependence on doping. Thus, in order to overcome this problem there is an urgent need to map the full two-dimensional phonon-dispersion relations around $K$ point in a similar manner as done routinely for electron energy dispersions by angle-resolved photoemission spectroscopy (ARPES).

\section{INELASTIC X-RAY SCATTERING AND AB INITIO CALCULATIONS}

In this paper we present a mapping of the detailed twodimensional (2D) phonon-dispersion relation around the $K$ point of graphite single crystals measured by IXS. This work constitutes the first application of $2 \mathrm{D}$ phonon mapping to graphite providing not only a correct assignment of the phonon branches and hence the phonon trigonal warping but also an all-experimental determination of the electron-phonon 

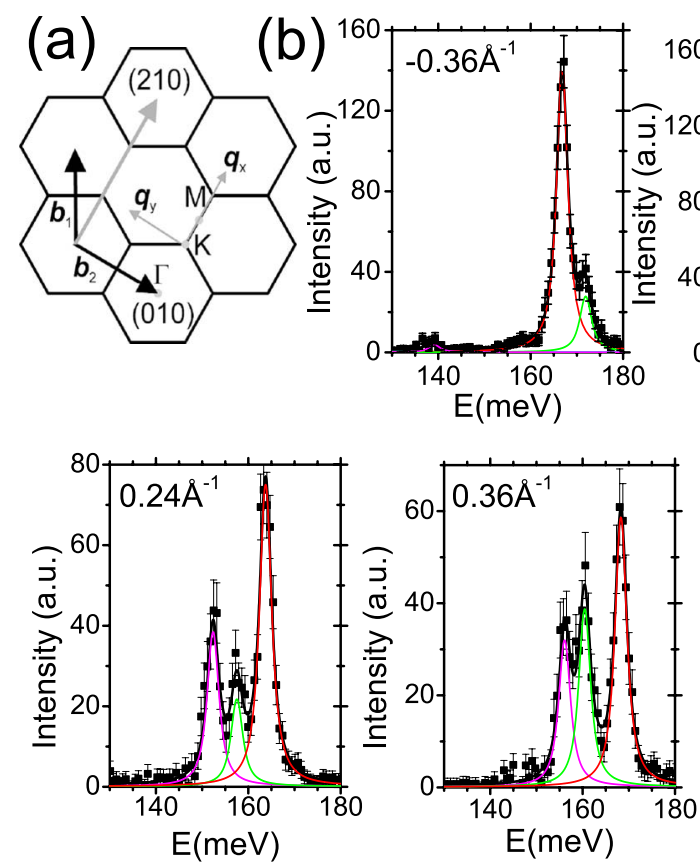

coupling. We expect that the technique of 2D phonon mapping is key for understanding phonon properties in a plethora of other materials, e.g., the high- $T_{c}$ superconductors. ${ }^{32}$

The phonon dispersion perpendicular to the graphene layers in graphite is practically zero for the phonon modes around $K$ (Refs. 11 and 33) and thus we can use graphite to test the phonons of graphene. We prove that at $K$, the two branches with LA and LO character and the branch with TO character are almost triply degenerate due to the Kohn anomaly which brings down the TO branch in energy. The phonon mode assignment is carried out by explicit comparison of the measured and calculated IXS intensities in the two-dimensional map and used to disentangle overlapping phonon modes. Using the present unambiguous assignment of the phonon branches and previously measured electron dispersions around $K$ we determine the value of the EPC entirely from experiments and find quantitative agreement to GW calculations.

The experiments were performed on beamline ID28 at the ESRF, utilizing the silicon (999) setup which yields monochromatized synchrotron light of $17794 \mathrm{eV}$, providing a total energy resolution of $\sim 3.3 \mathrm{meV} .{ }^{34}$ The beam size at the sample position was $60 \times 30 \mu \mathrm{m}^{2}$. As the spectrometer is equipped with nine analyzers, nine IXS spectra were recorded simultaneously, thus allowing an efficient coverage of the relevant portion of the phonon-dispersion branches. Single crystalline graphite samples with about $1 \mathrm{~cm}$ diameter and $100 \mu \mathrm{m}$ thickness were mounted on a goniometer. In Fig. 1(a) the scattering geometry and the coordinate system used throughout this work is shown. We collected IXS spectra along the line starting from the (010) reciprocal-lattice point in the (210) direction. We have purposely chosen this line since calculations of the IXS intensities reveal the highest intensity for the TO mode in the (010) BZ.

The phonon frequencies are the eigenvalues of $\sqrt{\mathcal{D}_{\mathbf{q}} / M}$, where $\mathcal{D}_{\mathbf{q}}$ is the dynamical matrix at the wave vector $\mathbf{q}$ and $M$ is the carbon mass. The DFT dynamical matrix $\mathcal{D}_{\mathbf{q}}^{\text {DFT }}$ is

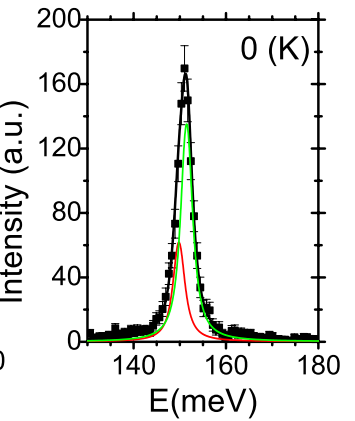

FIG. 1. (Color online) (a) Scattering geometry for the IXS experiments: spectra are collected along a line starting from the (010) BZ along the (210) (i.e., $\Gamma K M)$ direction. (b) IXS spectra of graphite single crystals along the $\Gamma K M$ line. The three phonon modes are fit by Lorentzians. The magenta and green Lorentzians denote the LA and LO modes. The TO mode is denoted by a red Lorentzian. The number in each viewgraph refers to the distance from $K$. (c) The calculated (left panel) and measured (right panel) IXS intensities for LO (green) and TO (red) modes. computed using linear response, ${ }^{1}$ with the exchange correlation of Ref. 35 and other computational details as in Ref. 14. The Kohn anomaly of the TO branch near $K$ is entirely determined $^{14,29}$ by the contribution (to $\mathcal{D}_{\mathbf{q}}$ ) of the phonon self-energy between $\pi$ bands $P_{\mathbf{q}}$ [defined in Eq. 1 of Ref. 29]. In particular, the TO branch corresponds to the eigenvector $\left|p_{\mathbf{q}}\right\rangle$ of $P_{\mathbf{q}}$ with the eigenvalue $p_{\mathbf{q}}$, that is, largest in modulo. $p_{\mathbf{q}}$ is proportional to the square of the EPC between the $\pi$ bands near the Fermi level. In Ref. 29 it was shown that DFT underestimates $\left|p_{\mathbf{q}}\right|$ and that $\left|p_{\mathbf{q}}\right|$ from the more accurate $\mathrm{GW}$ calculations is larger by a factor $r^{\mathrm{GW}}=1.61$. Here, following Ref. 29, we compute the GW dynamical matrices as

$$
\mathcal{D}_{\mathbf{q}}^{\mathrm{GW}}=\mathcal{D}_{\mathbf{q}}^{\mathrm{DFT}}+\left|p_{\mathbf{q}}\right\rangle\left\langle p_{\mathbf{q}}\right|\left\{\left(r^{\mathrm{GW}}-1\right) p_{\mathbf{q}}+\Delta\right\},
$$

where $\Delta$ is a constant independent of $\mathbf{q} \cdot r^{\mathrm{GW}}$ determines the slope of the TO branch near $K$. $\Delta$ shifts rigidly the TO branch with respect to the other branches. $\Delta$ cannot be computed easily from $a b$ initio calculations (see footnote 24 of Ref. 29) and is fitted to reproduce the present measurements. Furthermore DFT slightly overestimates (in a systematic manner) the phonon frequencies of the branches not affected by the Kohn anomaly, thus all the frequencies are scaled by $-1.3 \%$ to simplify the comparison with measurements.

Selected raw IXS spectra along the $\Gamma K M$ direction are shown in Fig. 1(b). These spectra contain the contributions of three phonon modes. A line-shape analysis using Lorentzians yields the position and intensity of each mode which are assigned to the LA, LO, and TO branches. Note that this characterization of phonon eigenvectors is only strictly valid for very small $k$ away from $\Gamma$ and we identify the phonon branches in the whole BZ according to their characterization close to $\Gamma .^{36}$ From symmetry considerations LO and LA modes are degenerate at the $K$ point. Our results clearly show that LO/LA and TO are almost degenerate at and close to the $K$ point. This is in striking contrast to previously determined 
(a)

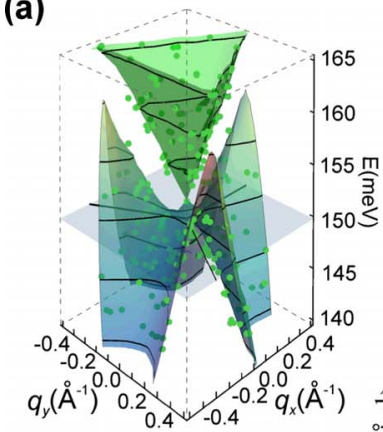

(b)

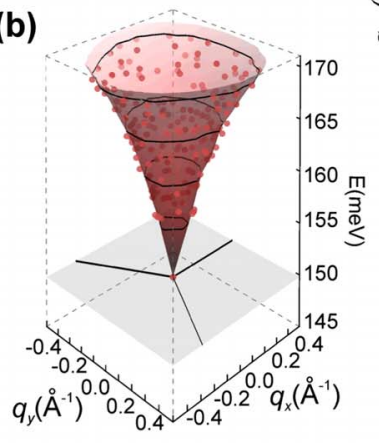

(c)

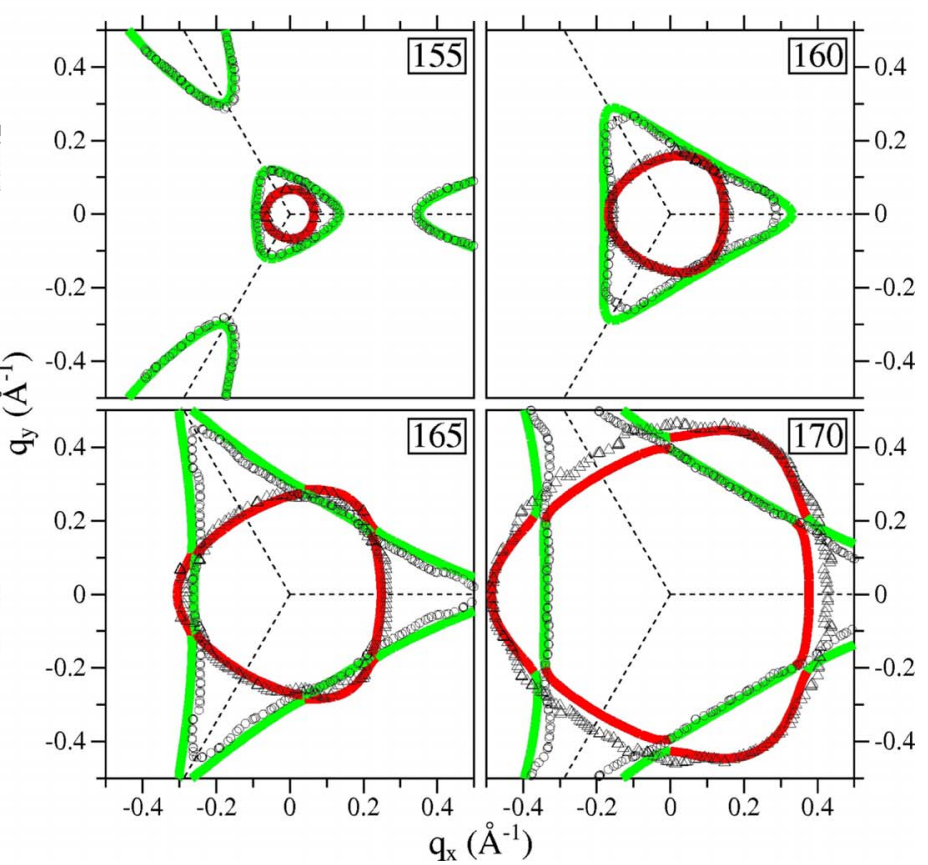

FIG. 2. (Color online) The interpolated experimental twodimensional phonon-dispersion relation for (a) the longitudinaloptic and acoustic-phonon branches and (b) the transverseoptical phonon branch that has a Kohn anomaly. Dots denote the experimental data taken from the maxima of Lorentzian fits [see Fig. 1(b)]. (c) Equienergy contours from the interpolated phonon surfaces for the three highest phonon branches around $K$ for energies $155-170 \mathrm{meV}$. $\bigcirc$ and $\triangle$ denote an interpolation to the raw experimental data in (a) and (b). Lines are GW calculations. The present color code follows the diabatic notation: branches with the same color have similar phonon patterns. phonon dispersions ${ }^{3,4}$ and cannot be explained by the standard DFT phonon calculations. ${ }^{3}$ Our mode assignment is based on a comparison of the measured and calculated IXS intensities as shown in Fig. 1(c) and further supported by a comparison to the GW-calculated phonon frequencies as shown later. It is clear that the calculated and experimental IXS intensities of LO and TO show the same pattern and thus we can safely assign the TO mode as the one with higher intensity along the considered path in the (010) BZ.

\section{TWO-DIMENSIONAL PHONON-DISPERSION RELATIONS}

From the maxima of the Lorentzians a set of $\sim 100$ data points is obtained in the $q_{x} q_{y}$ plane in a region of $\pm 0.6 \AA^{-1}$ around the $K$ point, which is further extended by the application of symmetry operations. In Figs. 2(a) and 2(b) the raw experimental data points around the $K$ point are shown. From these data points the three-dimensional surfaces of the phonon-dispersion relations are interpolated using spline functions. The interpolated phonon maps are used for further data analysis. The phonon trigonal warping effect for the LA, LO, and TO modes around the $K$ point is illustrated from the phonon equienergy contours shown in Fig. 2(c) along with the GW calculations. Notably, the experiments and calculations are in excellent agreement to each other which further supports the mode assignment performed above. Interestingly, the phonon trigonal warping effect of the TO mode is opposite to the electronic trigonal warping effect, i.e., the slope of the TO phonon branch is higher in $K M$ direction, whereas the electronic bands have a higher slope in $K \Gamma$ direction. ${ }^{30}$ For the LA and LO modes, the phonon trigonal warping has the same angular dependence as for the electronic bands. The fact that the equienergy contour of the TO mode is almost circular, is also in agreement with the angular dependence of the kink due to EPC in the quasiparticle dispersion measured by ARPES, where no change in the kink position, i.e., the energy of the coupling phonon ${ }^{37}$ was observed. Furthermore, the observed contour for the TO mode can explain why the Raman linewidth of the $G^{\prime}$ peak depends weakly on the exciting laser energy. The trigonal warping of electrons and the TO phonons is opposite, canceling the effects of enlarging/shrinking of the Raman linewidths. Furthermore it is clear that the LO and TO branches cross along the high-symmetry $K \Gamma$ direction but undergo anticrossing along all the other directions. In Fig. 3 we show the phonon dispersion along the high-symmetry lines and compare it to previous DFT calculations and our GW results. It can be observed that (1) the three branches are almost

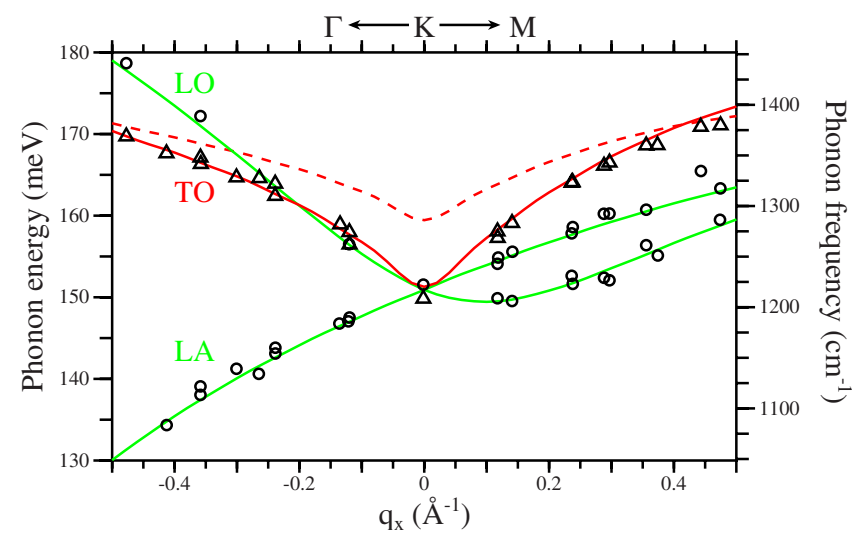

FIG. 3. (Color online) Phonon dispersion along the ГKM direction. Points are measurements extracted from the maxima of the Lorentzians from Fig. 1(b). Full lines are GW calculations. The dashed line is the TO branch from DFT calculations. DFT calculations of the longitudinal branches are not affected significantly by GW corrections and therefore not shown. The color code is the same as in Fig. 2. 
touching each other at $K,(2)$ the LO and TO branches cross each other in $K \Gamma$ direction, and (3) the proper inclusion of the electron-electron correlation (through the GW approach) in the calculation of the EPC is crucial to obtain a good agreement with the experiment. Indeed GW calculations are in almost perfect agreement with measurements while DFT calculations severely underestimate the phonon slope of the TO branch and thus the EPC at $K$ and overestimate the phonon energy. For precise description of the experimental phonon-dispersion relation, we provide simple fit formulas for phonon dispersions of the TO and LO branches. The TO phonon dispersion is given by

$$
E_{\mathrm{TO}}(q, \theta)=2.56 \cos (3 \theta) q^{2}-62.75 q^{2}+73.07 q+149.8
$$

and the LO phonon dispersion is best fit by

$$
\begin{aligned}
E_{\mathrm{LO}}(q, \theta)= & -10.25 q \cos ^{4}(3 \theta)-5.32 q \cos ^{3}(3 \theta) \\
& +5.47 q \cos ^{2}(3 \theta)+26.44 q^{2}+37.01 q \\
& -25.19 q^{2} \cos (3 \theta)+151.5
\end{aligned}
$$

Here $E_{\mathrm{TO}}(q)$ and $E_{\mathrm{LO}}(q)$ denote the phonon energies in meV of TO and LO branch, respectively, $q$ is the phonon wave vector measured in units $\AA^{-1}$ from $K$ point [see Fig. 1(a)] and $\theta$ the angle away from the $K M$ direction. From these fits we obtain the slope of the TO branch at $K, S_{\mathrm{TO}}^{K}=73.07 \mathrm{meV} \AA$, from which we can derive an experimental value for the square of the EPC constant between $\pi$ bands at $K,\left\langle D_{K}^{2}\right\rangle_{F}{ }^{14}$ Indeed, [see Eq. (10) of Ref. 14],

$$
\left\langle D_{K}^{2}\right\rangle_{F}=\frac{8 S_{\mathrm{TO}}^{K} M \hbar \omega_{\mathrm{TO}}^{K} v_{f}}{\sqrt{3} \hbar a_{0}^{2}},
$$

where $\left\langle D_{K}^{2}\right\rangle_{F}$ is defined in Ref. 29 and all the parameters on the right-hand side were experimentally determined in this work or previously by ARPES. ${ }^{30}$ In particular, $\hbar \omega_{\mathrm{TO}}^{K}$ $=149.8 \mathrm{meV}$ is the TO phonon energy at $K, v_{F}=1.05$ $\times 10^{6} \mathrm{~ms}^{-1}$ (Refs. 30 and 38 ) is the Fermi velocity, $M$ is the carbon atom mass, and $a_{0}=2.46 \AA$ is the in-plane lattice constant. Using Eq. (3), we obtain $\left\langle D_{K}^{2}\right\rangle_{F}=166(\mathrm{eV} / \AA)^{2}$ which is in excellent agreement to the theoretical $\mathrm{GW}$ value of $\left\langle D_{K}^{2}\right\rangle_{F}=164(\mathrm{eV} / \AA)^{2}$ (Ref. 29) and is to be compared to much lower DFT value of $\left\langle D_{K}^{2}\right\rangle_{F}=92(\mathrm{eV} / \AA)^{2} \cdot{ }^{29}$ Finally, concerning the temperature dependence of the energy ordering and the LO-TO difference at $K$, we performed experiments at three different temperatures $(300,150$, and $15 \mathrm{~K}$ ) and find no change in the ordering and a slight increase in the energy difference at $K$ with decreasing temperature $(2.2$ $\mathrm{meV}$ instead of $0.7 \mathrm{meV}$ ).

\section{SUMMARY}

In summary we have disentangled overlapping phonon branches and mapped the $2 \mathrm{D}$ phonon surfaces at the $K$ point of graphite using a combination of IXS and $a b$ initio calculations. Excellent agreement for the phonon energies was found once many-body effects at the GW level are considered. The three highest branches around $K$ point are almost triply degenerate, in stark contrast to previous measurements and DFT calculations. ${ }^{3,4}$ Furthermore the Kohn anomaly of the TO phonon branch at $K$ was directly measured by IXS and the EPC was determined entirely from experiments. Interestingly, this is the same phonon branch that is responsible for the kink in the ARPES measurements of doped graphene layers. Thus the present experiments provide a complementary view of EPC compared to our previous ARPES measurements, where we found a kink related to EPC at 166 meV. ${ }^{37}$ The good agreement between the two completely independent data sets strongly supports our phonon-mode assignment. The question which phonon branch is responsible for the kink in the ARPES can be answered by considering the phonon energy and its angular dependence around $K$, i.e., the phonon trigonal warping. Since both, the LO and TO branch have phonons with an energy of $166 \mathrm{meV}$ at $K$, the kink can, in principle, come from any of LO and TO modes (the LA branch does not have phonon with $166 \mathrm{meV}$ close to $K)$. However, when we consider the angular dependence of the kink position, we find that it is isotropic from the ARPES measurements and therefore it must come from a phonon branch with no or very little trigonal warping. This condition is clearly only met by the TO phonon branch. If the kink in the ARPES came from the LO phonon branch, the kink position would be different in $K \Gamma$ and in $K M$ directions. This assignment is also supported by the observation from double-resonance Raman, where EPC of electrons from the $\pi^{*}$ band to a $K$ point phonon is responsible for the large $G^{\prime}$ band intensity. In this case, the responsible phonon is also from the TO branch. We also found that the trigonal warping of the TO phonon branch is in the opposite direction as compared to the electronic trigonal warping and that of the LO phonon branch. This might be important for understanding the linewidth of the double-resonant Raman peaks.

\section{ACKNOWLEDGMENTS}

A.G. acknowledges an APART grant from the Austrian Academy of Sciences and a Marie Curie Reintegration grant (ECO-GRAPHENE) from the EU. J.S. acknowledges support from Spanish MICINN (Grants No. MAT2007-60087 and No. ENE2008-04373) and Generalitat de Catalunya (Grant No. 2005SGR00535). A.R. and L.W. are funded by Spanish MEC (Grant No. FIS2007-65702-C02-01), “Grupos Consolidados UPV/EHU and DIPC del Gobierno Vasco" (IT319-07) and EC-I3 ETSF project (Contract No. 211956). Calculations were done at IDRIS and Mare Nostrum "Red Espanola de Supercomputacion.” A.G. and T.P. acknowledge the EU for travel support for their stay at the ESRF. 
${ }^{1}$ S. Baroni, S. de Gironcoli, A. Dal Corso, and P. Giannozzi, Rev. Mod. Phys. 73, 515 (2001).

${ }^{2}$ R. Al-Jishi and G. Dresselhaus, Phys. Rev. B 26, 4514 (1982).

${ }^{3}$ J. Maultzsch, S. Reich, C. Thomsen, H. Requardt, and P. Ordejon, Phys. Rev. Lett. 92, 075501 (2004).

${ }^{4}$ M. Mohr, J. Maultzsch, E. Dobardžić, S. Reich, I. Milošević, M. Damnjanović, A. Bosak, M. Krisch, and C. Thomsen, Phys. Rev. B 76, 035439 (2007).

${ }^{5}$ R. Saito, A. Jorio, A. G. Souza Filho, G. Dresselhaus, M. S. Dresselhaus, and M. A. Pimenta, Phys. Rev. Lett. 88, 027401 (2001).

${ }^{6}$ Ge. G. Samsonidze, R. Saito, A. Jorio, A. G. Souza Filho, A. Grüneis, M. A. Pimenta, G. Dresselhaus, and M. S. Dresselhaus, Phys. Rev. Lett. 90, 027403 (2003).

${ }^{7}$ T. Aizawa, R. Souda, S. Otani, Y. Ishizawa, and C. Oshima, Phys. Rev. B 42, 11469 (1990).

${ }^{8}$ G. Kresse, J. Furthmüller, and J. Hafner, Europhys. Lett. 32, 729 (1995).

${ }^{9}$ P. Pavone, R. Bauer, K. Karch, O. Schütt, S. Vent, W. Windl, D. Strauch, S. Baroni, and S. de Gironcoli, Physica B (Amsterdam) 219\&220, 439 (1996).

${ }^{10}$ A. Bosak, M. Krisch, M. Mohr, J. Maultzsch, and C. Thomsen, Phys. Rev. B 75, 153408 (2007).

${ }^{11}$ L. Wirtz and A. Rubio, Solid State Commun. 131, 141 (2004).

${ }^{12}$ E. J. Woll and W. Kohn, Phys. Rev. 126, 1693 (1962).

${ }^{13}$ T. Ando, J. Phys. Soc. Jpn. 75, 124701 (2006).

${ }^{14}$ S. Piscanec, M. Lazzeri, F. Mauri, A. C. Ferrari, and J. Robertson, Phys. Rev. Lett. 93, 185503 (2004).

${ }^{15}$ M. Lazzeri and F. Mauri, Phys. Rev. Lett. 97, 266407 (2006).

${ }^{16}$ S. Pisana, M. Lazzeri, C. Casiraghi, K. S. Novoselov, A. K. Geim, A. C. Ferrari, and F. Mauri, Nature Mater. 6, 198 (2007).

${ }^{17}$ J. Yan, Y. Zhang, P. Kim, and A. Pinczuk, Phys. Rev. Lett. 98, 166802 (2007).

${ }^{18}$ N. Caudal, A. M. Saitta, M. Lazzeri, and F. Mauri, Phys. Rev. B 75, 115423 (2007).

${ }^{19}$ A. Das, A. K. Sood, A. Govindaraj, A. M. Saitta, M. Lazzeri, F. Mauri, and C. N. R Rao, Phys. Rev. Lett. 99, 136803 (2007).

${ }^{20}$ H. Farhat, H. Son, Ge. G. Samsonidze, S. Reich, M. S. Dressel- haus, and J. Kong, Phys. Rev. Lett. 99, 145506 (2007).

${ }^{21}$ J. C. Tsang, M. Freitag, V. Perebeinos, J. Liu, and Ph. Avouris, Nat. Nanotechnol. 2, 725 (2007).

${ }^{22}$ K. I. Sasaki, R. Saito, G. Dresselhaus, M. S. Dresselhaus, H. Farhat, and J. Kong, Phys. Rev. B 78, 235405 (2008).

${ }^{23}$ N. B. Hannay, T. H. Geballe, B. T. Matthias, K. Andres, P. Schmidt, and D. MacNair, Phys. Rev. Lett. 14, 225 (1965).

${ }^{24}$ M. Calandra and F. Mauri, Phys. Rev. Lett. 95, 237002 (2005).

${ }^{25}$ C. Thomsen and S. Reich, Phys. Rev. Lett. 85, 5214 (2000).

${ }^{26}$ A. C. Ferrari, J. C. Meyer, V. Scardaci, C. Casiraghi, M. Lazzeri, F. Mauri, S. Piscanec, D. Jiang, K. S. Novoselov, S. Roth, and A. K. Geim, Phys. Rev. Lett. 97, 187401 (2006).

${ }^{27}$ D. Graf, F. Molitor, K. Ensslin, C. Stampfer, A. Jungen, C. Hierold, and L. Wirtz, Nano Lett. 7, 238 (2007).

${ }^{28}$ D. M. Basko and I. L. Aleiner, Phys. Rev. B 77, 041409(R) (2008).

${ }^{29}$ M. Lazzeri, C. Attaccalite, L. Wirtz, and F. Mauri, Phys. Rev. B 78, 081406(R) (2008).

${ }^{30}$ A. Grüneis, C. Attaccalite, T. Pichler, V. Zabolotnyy, H. Shiozawa, S. L. Molodtsov, D. Inosov, A. Koitzsch, M. Knupfer, J. Schiessling, R. Follath, R. Weber, P. Rudolf, L. Wirtz, and A. Rubio, Phys. Rev. Lett. 100, 037601 (2008).

${ }^{31}$ Michele Lazzeri, S. Piscanec, Francesco Mauri, A. C. Ferrari, and J. Robertson, Phys. Rev. B 73, 155426 (2006).

${ }^{32}$ J. Graf, M. d'Astuto, C. Jozwiak, D. R. Garcia, N. L. Saini, M. Krisch, K. Ikeuchi, A. Q. R. Baron, H. Eisaki, and A. Lanzara, Phys. Rev. Lett. 100, 227002 (2008).

${ }^{33}$ N. Mounet and N. Marzari, Phys. Rev. B 71, 205214 (2005).

${ }^{34}$ M. Krisch, J. Raman Spectrosc. 34, 628 (2003).

${ }^{35}$ J. P. Perdew, K. Burke, and M. Ernzerhof, Phys. Rev. Lett. 77, 3865 (1996).

${ }^{36}$ N. Bonini, M. Lazzeri, N. Marzari, and F. Mauri, Phys. Rev. Lett. 99, 176802 (2007).

${ }^{37}$ A. Grüneis, C. Attaccalite, A. Rubio, D. V. Vyalikh, S. L. Molodtsov, J. Fink, R. Follath, W. Eberhardt, B. Büchner, and T. Pichler, Phys. Rev. B 79, 205106 (2009).

${ }^{38}$ A. Grüneis, C. Attaccalite, L. Wirtz, H. Shiozawa, R. Saito, T. Pichler, and A. Rubio, Phys. Rev. B 78, 205425 (2008). 